Elsevier required licence: (c) $<2018>$. This manuscript version is made available under the CC-BY-NC-ND 4.0 license http://creativecommons.org/licenses/bync-nd/4.0/ 
An online resource to promote vocational interests among job-seekers with multiple sclerosis: a randomized controlled trial in Australia

Dorstyn, D., Roberts, R.M., Murphy, G., Kneebone, I., Craig, A., \& Migliorini, C. (in press). An online resource to promote vocational interests among job-seekers with multiple sclerosis: A randomized controlled trial in Australia. Archives of Physical Medicine and Rehabilitation. doi:10.1016/j.apmr.2017.08.475

This a final submitted copy however it may differ from the published version which is available from the journal website

University of Adelaide Human Research Ethics Protocol No. H-2016-019

Diana Dorstyn PhD, ${ }^{1}$ Rachel Roberts PhD, ${ }^{1}$ Gregory Murphy PhD, ${ }^{2}$ lan Kneebone PsychD, ${ }^{3}$ Ashley Craig PhD, ${ }^{4}$ Christine Migliorini PhD ${ }^{5}$

${ }^{1}$ Faculty of Health and Medical Sciences, School of Psychology, University of Adelaide, South Australia, Australia

${ }^{2}$ School of Public Health, LaTrobe University, Victoria, Australia

${ }^{3}$ Discipline of Clinical Psychology, Graduate School of Health, University of Technology Sydney, New South Wales, Australia

${ }^{4}$ Sydney Medical School-Northern, Kolling Institute of Medical Research, The University of Sydney, New South Wales, Australia

${ }^{5}$ Department of Occupational Therapy, School of Primary Health Care, Monash University, Victoria, Australia

Corresponding author: Diana Dorstyn, School of Psychology, University of Adelaide, North 
Terrace, Adelaide SA 5005, Ph: +61 88313 0649, Email: diana.dorstyn@adelaide.edu.au

Acknowledgements: The authors are very grateful for the cooperation of participants who dedicated their time to this study and to the community organisations who kindly assisted with recruitment. We also wish to acknowledge R. Haub and E. Potter, School of Psychology, University of Adelaide, for assistance with data collection, and Dr S. Howell, Senior Statistician, of Public Health, University of Adelaide, for statistical advice.

Financial Disclosure. We certify that no party having a direct interest in the results of the research supporting this article has or will confer a benefit on us or on any organization with which we are associated.

Reprints will be available from the authors.

This study is registered with MS Research Australia Clinical Trials Network and the Australian and New Zealand Clinical Trials Registry (ACTRN12617000728336)

\section{An online resource to promote vocational interests among job-seekers with multiple sclerosis: a randomized controlled trial in Australia}

\footnotetext{
Abstract

Objective. To provide a preliminary evaluation of the effectiveness of an on-line resource for job-seekers with multiple sclerosis.

Design. Randomized controlled design.

Setting. Community-dwelling cohort in Australia.
} 
Participants. Forty-seven adults with relapsing-remitting or progressive MS accessed an emaildelivered, 7-module resource - 'Work and MS' - over a 4-week period. Fifty wait-list control participants were offered the opportunity to access 'Work and MS' 4 weeks post-enrolment. Main Outcome Measures. Primary outcomes focused on vocational interests (My Vocational Situation Scale; MVS) and self-efficacy in job-seeking activities (Job-Procurement Self Efficacy Scale; JSES). Secondary outcomes focused on perceived workplace difficulties (Multiple Sclerosis Work Difficulties Questionnaire; MSWDQ), optimism (Life Orientation Test Revised; LOT-R), and mood (Patient Health Questionnaire-9; PHQ-9).

Results. ITT analyses revealed pre-post gains: participants who accessed 'Work and MS' reported improved confidence in their career goals (MVS $g=0.55$ [CI: 0.14, 0.96] $P=0.008$ ) and positively re-appraised potential workplace difficulties (MSWDQ $g$ range: 0.42 to $0.47 ; P$ range $=$ 0.023 to 0.042 ). The effect on job self-efficacy was not significant, although changed in the expected direction ( $g=0.17$, [CI: $-0.23,0.57] P=0.409$ ). Completer data revealed larger, significant effect estimates ( $g$ range $=0.52$ to $0.64, P$ range $=0.009$ to 0.035 ).

Conclusions. Findings provide preliminary support for the utility of a job-information resource, 'Work and MS', to augment existing employment services. The results also suggest the need to test employment-ready interventions in a larger study population. This might include the addition of on-line peer support to increase intervention compliance.

Keywords: multiple sclerosis, employment, unemployment, internet, rehabilitation, return-towork

\section{Abbreviations}

JSES Job-Procurement Self Efficacy Scale 
MS multiple sclerosis

MSRS-R Multiple Sclerosis Rating Scale-Revised

MSWDQ Multiple Sclerosis Work Difficulties Questionnaire

MVS My Vocational Situation Scale

LOT-R Life Orientation Test - Revised

PHQ-9 Patient Health Questionnaire-9

SOS Service Obstacles Scale

Access to employment for those with a disability is a complex socioeconomic problem. This is particularly so in Australia, where performance in the employment of people with a disability compares poorly with that of the United Kingdom and Europe: in 2012, 40\% of people with a disability were employed in Australia. (1) This is despite national investment into services aimed at increasing the numbers of employees working while living with a disability. $(2,3)$ The problem is certainly highlighted by the high social and economic costs associated with unemployment for those with a chronic neurological condition such as multiple sclerosis (MS), which typically affects young adults in their most productive years. (4) Loss of wages equate to an estimated \$AUD 494 million: $47 \%$ of the total costs of MS management in Australia. (5)

A complex social construct such as employment is best understood from a biopsychosocial framework. One such framework is the International Classification of Functioning, Disability and Health (ICF), according to which biological, socio-environmental and personal factors interact to create or eliminate disability. $(6,7)$ Studies confirm the associations between older age, greater reported disability, longer disease duration, MS-related cognitive difficulties, and employment loss. (8-11) Persons with a disability have also identified attitudinal, 
policy and programmatic barriers as contributing to early retirement from the Australian labor force (e.g. inflexible working practices, unsupportive work culture, inaccessible work environment; 2,12). Telerehabilitation - the use of information and communication technologies as a medium for the provision of rehabilitation services - may help to circumvent some of these aforementioned barriers. However, controlled trials are needed to guide treating clinicians on the validity, reliability and efficiency of telerehabilitation services designed to enhance vocational achievement. (13-16)

Employment should also be considered in tandem with job-related psychological factors, such as vocational identity. $(6,17,18)$ For persons with MS, this means an ability to work effectively and make sensible career decisions in the event of unforeseen MS-related problems. A sense of self-efficacy and control over career decisions, alongside perceptions of fewer unmanageable workplace barriers and positive expectations about employment, may also contribute to a more integrated sense of vocational identity for those with MS. $(1317,18)$ Conversely, an inability to make career decisions is associated with lowered life satisfaction, pessimism and subjective wellbeing among the general population, $(20,21)$ although this requires further exploration in persons with MS.

In sum, strategies are needed to strengthen Australian vocational care for persons with MS. To this end, a consumer-based job information resource, 'Work and MS', was developed and piloted by our research team. (13) 'Work and MS' is strengths-based: the aim is to help jobseekers consider work options that best match their skills, abilities and interests and, in turn, contribute to a biopsychosocial employment approach. Early results from the use of 'Work and MS' have been promising: the feasibility of recruiting participants to engage in the resource was ascertained and moderate to large improvements in vocational identity and self-efficacy reported. 
(13) The objective of the current study was to confirm these findings using a randomized controlled design. It was anticipated that access to 'Work and MS' would facilitate positive job identity in comparison to a no-intervention (wait-list) control condition. Of secondary interest was whether 'Work and MS' might improve self-efficacy, expectations of work difficulties, and general mental health (i.e. optimism, mood).

\section{Methods}

Eligibility criteria.

Community-dwelling adults (aged $\geq 18$ years) with MS actively seeking employment were eligible to participate. This included persons wanting to return to the workforce in addition to those currently employed yet seeking alternative work. Although this represented a heterogeneous job-seeker group, with potentially different experiences and perspectives in relation to RTW, both seeking and maintaining suitable employment are equally problematic for Australian adults with a disability. $(2,12)$ English fluency and access to a computer with an Internet provider were additional requirements. Retirees were excluded, as the focus was on individuals considered currently employable (i.e. those with the capacity to work). Participants were consecutively recruited over a 4-month period (May-Aug, 2016). Data for 16 participants were removed (i.e. $>80 \%$ of baseline survey incomplete, including primary outcome). An additional two participants requested to withdraw from the study whilst two participants who identified as 'medically retired' were ineligible, resulting in a final sample of 95 at study commencement.

Procedure. 
Following project approval by the University of Adelaide Human Research Ethics Committee (project no. H-2016-019), social networking sites $(22,23,24)$ of community MS agencies (CoAct/MS Employment Support Service, MS Research Australia, MS Society of Australia, MS Society of South Australia and Northern Territory) were approached to assist with recruitment.

Participants were randomly assigned to the 'Work and MS' intervention or wait-list control group on enrolment, using Survey Monkey’s ${ }^{\mathrm{TM}}$ A/B test randomization feature. Group assignment was not blinded: both participants and the lead researcher (D.D) were aware of group assignment. All participants completed an online survey on enrolment (Time 1). Following this, intervention participants were emailed the first three 'Work and MS' modules to review at their own pace. A follow-up email with the remaining 4 modules was sent one week later. At 4 weeks post-enrolment (Time 2) all participants received a link to a follow-up online survey. Reminders for survey completion were subsequently sent on a weekly basis over the next month. Following completion of this second assessment, control participants were emailed the 'Work and MS' resource. See CONSORT flow chart for details of participant recruitment (Figure 1).

Intervention.

Developed in consultation with consumers with MS (13), 'Work and MS' comprises of one introductory and six learning modules. The modules focus on requisite job skills sets, namely: the job-seeking process (3 modules), job-interviewing skills (2 modules), and career development (1 module; see Table 1). Each module, presented as a PowerPoint presentation to maximize readability, includes the following key components: (1) module objectives; (2) interactive components to illustrate key points (e.g. on-line activity worksheets that can be completed and emailed to moderator for feedback); (3) downloadable education materials, and (4) a summary of 
content. Case examples, sourced from participants involved in our pilot trial (13) were additionally incorporated to illustrate practical aspects of return-to-work. 'Work and MS' adopts a self-management approach, with participants progressing through the modules independently and at their own pace. The modules were designed to be brief, with pilot testing suggesting that each requires up to 20 minutes to complete online (excluding activity worksheets).

Baseline measures.

In addition to socio-demographic information (age, sex, relationship, employment, education), the Multiple Sclerosis Self-Rating Scale (MSRS-R) (25) was administered as a measure of functional disability. The MSRS, adapted from Guy’s Neurological Disability Scale, $(26,27)$ assesses impairment across eight domains: walking, upper limb function, bladder/bowel problems, cognition, speech, swallowing, vision, and pain. Items are rated from 0 (“none”) to 4 ("severe") and totaled. In this study, the MSRS-R had acceptable internal consistency ( $\alpha=0.73$ ). Satisfaction with available employment support services was examined using an adapted version of the Service Obstacles Scale (SOS) (28) (i.e. references to 'brain injury’ replaced with 'multiple sclerosis'). As per the original SOS, items were rated on a 7-point Likert-type scale ranging from Strongly Disagree through Strongly Agree, with inter-item correlations being suitably related $(\alpha=0.79)$.

Primary outcomes.

Participants completed five standardized self-report measures on enrolment (Time 1) and 4 weeks post (Time 2). This included the vocational identity subscale from Holland, Gottfredson and Power's (29) My Vocational Situation Scale (MVS), to examine vocational goals and 
interests (e.g. "I don't know what my work-related strengths and weaknesses are”). Consistent with previous psychometric reviews $(29,30)$, acceptable Cronbach alphas (Time $1=0.84$, Time 2 $=0.86$ ) were demonstrated in this study.

Self-confidence in relation to seeking work was evaluated with the 25-item JobProcurement Self-efficacy Scale (JSES, 31). Based on Bandura’s (32) Social Learning Theory, the JSES measures perceived ability to perform the skills involved in seeking employment (e.g. " $I$ know how to find services in the community that could help me find a job"). The JSES demonstrated excellent reliability in this study (Time 1 and $2 \alpha=0.94$ ).

Secondary outcomes.

The frequency of experienced workplace difficulties was evaluated using the Multiple Sclerosis Work Difficulties Questionnaire (MSWDQ, 33), with sub-scale scores computed as a percentage to enable cross-comparison. Only the six subscales directly targeted by the 'Work and MS’ intervention were utilized: work home balance difficulties, low self-esteem, interpersonal difficulties, workplace inaccessibility, financial security concerns, and non-supportive workplace. Consistent with Honan et al’s (33) validation study, all subscales demonstrated acceptable internal consistency ( $\alpha$ range: 0.71-0.92). Optimism was evaluated with the Life Orientation Test -Revised (LOT-R; 34,35) and depressed mood with the 9-item Patient Health Questionnaire (3639). High internal consistency was demonstrated at each assessment point for both measures ( $\alpha$ range $=0.86-0.93)$.

Statistical analyses. 
Data were analyzed using the Statistical Package for the Social Sciences (IBM-SPSS v24, Armonk, New York). Descriptive statistics provided a summary of the sample characteristics. The comparability of intervention $(n=45)$ and wait-list control $(n=50)$ participants in relation to demographics (i.e. age, gender), MS characteristics (MSRS-R, MS subtype, time since diagnosis) and baseline psychological measurements (MVS, JSES, MSWDQ, LOT-R, PHQ-9) was assessed using independent samples $t$-tests (continuous data) and chi-square analyses (categorical data). Differences between employed $(n=72)$ and not employed persons with MS ( $n=23)$, were also examined. Patterns in missing data were explored by examining group differences between participants who completed their pre- and post-surveys $(n=61)$ and those with incomplete data $(n$ $=34)$.

Pearson correlation coefficients were utilized to determine the association between each outcome and key contextual variables - age, time since diagnosis, functioning (MSRS-R), and service satisfaction (SOS). Correlational analyses were also conducted to examine the degree to which there was or statistical overlap between the primary and secondary outcomes.

Group differences ('Work and MS' vs Controls) on all outcomes were assessed using independent $t$-tests on difference scores (T2-T1). An intention-to-treat analysis was performed using a conservative last observation: effectively assuming that those lost to followup (i.e. T2 assessment) had unchanged outcomes. (40) All group comparisons were supplemented with a standardized index, Hedges' $g(41)$, with values of $0.2,0.5$ and 0.8 equating to small, medium and large group differences, respectively. (42)

A statistical power analysis was performed for sample size calculation. The projected sample size needed to detect a large effect of 0.80 (using Cohen's criteria (42) and based on our pilot findings (13), with an alpha level of $p<0.05$ and power $=0.80$ for a two-tailed independent 
samples $t$-test, is approximately 52 (or 26 per group) (43). Thus, our sample size of 95 was adequate to address the study’s main objective.

\section{Results}

Participant characteristics.

Ninety-five persons with MS, averaging 42 years of age, comprised the final sample (Table 2). The female predominance (5:1, female:male) is consistent with Australia data on MS prevalence. (44) Self-reported MS details included a relapsing-remitting course, the most common disease subtype, (45) associated with varying degrees of physical impairment. Most ( $n=73$, 77\%) were currently working on a full-, part-time or casual basis although seeking alternative employment. Mean SOS ratings $(18.97, \mathrm{SD}=5.08$, range $=7-28$ ) indicated that participants were ambivalent about utilizing employment support services, with 55\% $(n=52)$ expressing dissatisfaction with service availability and/or quality. The majority $(68 \%, n=65)$ also acknowledged having limited knowledge and information about the types of employment services available in their local area. Most participants (74\%, $n=70)$ self-reported symptoms of clinical depression at baseline (Time 1 PHQ-9 mild [5-9] = 36\%; moderate (10-14) 17\%; moderately severe (15-19) 14\%; severe (>19) $7 \%)$.

Adherence to 'Work and MS' was acceptable: 69\% ( $n=31)$ indicated having accessed the material (Figure 1). Intervention and wait-list control participants were comparable in age, functioning (MSRS-R), and psychological characteristics (Table 2). Similarly, baseline characteristics of the two job-seeker groups targeted (i.e. those employed but seeking alternative work vs. those currently not employed) did not significantly differ. Although withdrawals cited fewer workplace barriers, including the quality of collegial relationships, in comparison to those 
who completed the study $(t(93)=-2.59, g=-0.67$ [CI: $-1.19,-0.17], P=0.01)$, no other significant differences (i.e. age, MSRS-R ratings, time since diagnosis, outcomes) were noted between these two groups.

Inter-variable relationships.

There was a significant effect of age for optimism: older adults had a more optimistic outlook than their younger counterparts (Table 3). Time since diagnosis, severity ratings (MSRS-R) and service dissatisfaction (SOS) were also associated with key outcomes. Those with recent onset MS readily identified workplace (i.e. financial) barriers. Similarly, those reporting greater MSrelated impairment and those who expressed dissatisfaction with the quality and/or accessibility of available employment supports experienced poor psychological functioning (MVS, PHQ-9) alongside workplace difficulties.

With the exception of the MSWDQ work-home balance and accessibility subscales, all of the primary and secondary outcomes were inter-correlated (see Table 3). The small to medium relationships suggest that these variables were not tapping entirely different constructs.

\section{Primary outcomes.}

Immediately after accessing 'Work and MS', participants reported a better understanding of their vocational and career goals (MVS $g$ : 0.55 [CI: 0.14, 0.96] $P=0.008$ ). This effect was larger for those who completed the study (Table 4). Although no significant group differences in job procurement self-efficacy were reported, the small and positive effect was in the expected direction, that is self-efficacy was enhanced. 


\section{Secondary outcomes.}

ITT data revealed medium and significant effect estimates in relation to perceived work difficulties: those who accessed 'Work and MS' reported improved expectations in relation to the impact of MS symptoms on general self-esteem ( $g$ : 0.47 [CI: $0.06,0.88$ ], $P=0.02)$, work relationships ( $g$ : 0.46 [CI: $0.07,0.86$ ], $P=0.03$ ); and financial concerns ( $g$ : 0.42 [CI: 0.02, 0.82], $P=0.04)$. Similar effects were noted when examining the data for intervention completers only.

\section{(Table 4)}

\section{Discussion}

The current results provide preliminary support for the utility of an email-based resource targeted to the needs of job-seekers with chronic MS. Free and accessible information on MS employment-related matters is important for this cohort. This includes a need to review/recognize one’s transferable job skills and interests and workplace solutions for issues directly or indirectly related to one’s MS). Practical resources targeted at job-seeking and job retention skills and knowledge (e.g. how to find a job and/or be identified by employers, preparing to job network) are also critical. The findings also highlight the need for rehabilitation and vocational counsellors to understand the complex psychological factors, such as work identity and self-efficacy perceptions, that may influence work participation when designing employment service programs.

A key strength of the 'Work and MS' intervention is its targeted focus to the needs of persons with MS (e.g. clarification of functional limitations, self-disclosure of MS) whilst also promoting skills acquisition (i.e. recognizing one’s transferable vocational skills and interests alongside job-seeking and job-networking skills). This type of biopsychosocial, health-specific 
approach is crucial for effective, sustainable job acquisition by persons with MS, yet is rarely considered in the context of vocational rehabilitation counselling. (47) Our focus on career maintenance and development is also novel, with vocational interventions traditionally centering on 'static' job placement. (48)

The results also suggest that more can be done to improve participation and engagement with a program like 'Work and MS'. Vocational interventions that incorporate ongoing support, monitoring and peer-sharing are highly regarded by adult job-seekers with a physical disability. $(49,50,51)$ This might include telecommunication-based supports to promote social networking and mentoring. (15) Future research is needed to test an improved 'Work and MS' intervention, by adding on-line moderator and peer support or by supplementing 'Work and MS' with direct comprehensive intervention (i.e. job-seeking skills training; 48). Sub-group analyses could then examine social support as a modifiable factor. Notably, 'Work and MS' was not designed as a stand-alone return-to-work intervention but, rather, a consumer-based resource that could be readily available to persons with MS as early as needed to support their vocational rehabilitation. Study limitations and future research.

A key methodological issue involves the attrition rate, and resulting low statistical power, for the secondary outcomes examined. Notably, online studies are characterized by poor adherence. (52) Nonetheless, further large-scale trials are needed to confirm the current findings. This should include an attention control group to determine the specific effects of 'Work and MS', in addition to testing for placebo effects. (53) Future trials might also host the 'Work and MS' resource on an online platform, to allow tracking of website traffic patterns. This type of data is critical in determining intervention effectiveness. Indeed, the disability literature suggests that whilst self-management education programs may lead to small, short- 
term improvements in psychological functioning, including self-efficacy, there remains little empirical data examining participation and successful completion. (54).

Future trials might also exclusively focus on unemployed persons who are looking for work, and exclude working participants. Although the two subgroups did not significantly differ on baseline characteristics in this study, these analyses were based on a limited dataset (i.e. 72 employed, 23 not employed). The lack of follow-up assessment is an additional limitation. It is not known whether the reported gains in vocational identity might be maintained over time and, in addition, whether they translate to behavioral outcomes. For example, future research might track participants' engagement with employment service organizations and the labor market.

There is, arguably, also a need to address environmental variables in the employment of persons with MS; an aspect not specifically examined by 'Work and MS'. Discriminatory hiring practices and inflexible workplace accommodations continue to be identified by job-seekers with a disability $(46,49,51,55,56)$. To ensure a holistic, biopsychosocial approach to vocational rehabilitation, 'Work and MS' could, potentially, be adapted and trialed as a resource package for employers and employees alike.

Criticisms and usefulness of the multidimensional psychological constructs explored in this review also need to be considered. Job identity and self-efficacy require further investigation with persons with MS. Importantly, the psychometric properties of the MVS and JSES were acceptable in this study and may serve as a foundation for further research, including longitudinal study designs to explore temporality. Other scales evaluating factors of potential importance in vocational rehabilitation of persons with MS have also been published. $(57,58)$

\section{Conclusion}


This controlled trial provides much needed data on the effectiveness of a targeted intervention for promoting job-seeking and work-ready skills and, potentially, reducing psychosocial barriers to RTW for persons with MS. Further comparative effectiveness trials will help to confirm the viability of 'Work and MS'. 


\section{Acknowledgements}

3 The authors are very grateful for the cooperation of participants, who dedicated their time to

4 this study, and to the community organizations who kindly assisted with recruitment. We also

5 wish to acknowledge R. Haub and E. Potter, from the School of Psychology, University of

6 Adelaide, for assistance with data collection, and Dr S. Howell, Senior Statistician from the

7 School of Public Health, University of Adelaide, for statistical advice.

8

\section{Declaration of interest}

10 The authors declare no conflicts of interest. 


\section{References}

1. Organisation for Economic Co-operation and Development. Connecting people with jobs: Key issues for raising labour market participation in Australia. Paris: OECD Publishing; 2017.

2. Copas S, Dorstyn D. Needs, challenges and goals of Australian women with disabilities: A preliminary survey. AJRC 2014; 20: 28-37.

3. Australian Human Rights Commission. Willing to work: National inquiry into employment discrimination against older Australians and Australians with disability. Australian Human Rights Commission, Sydney: 2016.

4. Howard J, Trevick S, Younger DS. Epidemiology of multiple sclerosis. Neurol Clin 2016; 34(4): 919-939.

5. Covance PTY LTD, Palmer A. Economic impact of multiple sclerosis in 2010. Australian MS longitudinal Sstudy. Sydney: Multiple Sclerosis Research Australia; 2011

6. World Health Organization. Towards a common language for functioning, disability and health: ICF. International Classification of Functioning, Disability and Health (p. 9). Geneva, Switzerland: WHO; 2002.

7. Frain M P, Bishop M, Rumrill PD, Chan F, Tansey TN, Strauser D, Chiu C-Y. Multiple sclerosis and employment: A research review based on the International Classification of Function. Rehab Rese Policy Educ 2015; 29: 153-164.

8. Bøe LHM, Telstad W, Grytten N, Kyte L, Aarseth J, Myhr KM, Bø L. Employment among patients with multiple sclerosis: A population study. PLoS One 2014; 9: e103317. 9. Honan CA, Brown RF, Batchelor J. Perceived cognitive difficulties and cognitive test 
Vocational rehabilitation and multiple sclerosis

performance as predictors of employment outcomes in people with multiple sclerosis. J Int Neuropsychol Soc 2015; 21: 156-168.

10. Julian L, Vella L, Vollmer T, Hadjimichael O, Mohr DC. Employment in multiple sclerosis. J Neurol 2008; 255: 1354-1360.

11. Roessler RT, Rumrill, PD, Fitzgerald SM. Predictors of employment status for people with multiple sclerosis. Rehabil Couns Bull 2004; 47: 96-103.

12. Ruhindwa A, Randall C, Cartmel J. Exploring the challenges experienced by people with disabilities in the employment sector in Australia: Advocating for inclusive practice - a review of literature. JoSI 2016; 7: 4-19.

13 . Dorstyn D, Roberts R, Murphy G, Kneebone I, Migliorini C, Craig A, et al. Piloting an email-based resource package for job seekers with multiple sclerosis. Disabil Rehabil 2016; 39: 867-873.

14. Khan F, Amatya B, Kesselring J. Telerehabilitation for persons with multiple sclerosis (Protocol). Cochrane Database Syst Rev 2013; 5: Art. No: CD010508.

15. Schmeler MR, Schein RM, McCue M, Betz K. Telerehabilitation clinical and vocational applications for assistive technology: Research, opportunities and challenges. Int J Telerehab 2009; 1: 59-72.

16. Sweetland J, Howse E, Playford D. A systematic review of research undertaken in vocational rehabilitation for people with multiple sclerosis. Disabil Rehabil 2012; 34: 2031-2038. 17. Escorpizo R. In Editors: Escorpizo R, Brage S, Homa D, Stucki G. (Eds.) 2015. Handbook of vocational rehabilitation and disability evaluation. Application and implementation of the ICF 
18. Skorikov VB, Vondracek FW. Occupational identity. In: Schwartz SJ, Luyckx K, Vignoles VL editors. Handbook of identity theory and research. New York: Springer Science and Business Media; 2011. P. 693-714.

19 Dorstyn D, Roberts R, Murphy G. Haub R. Psychological benefits of employment for adults with multiple sclerosis: A systematic review and meta-analysis. J Health Psychol 2017. Advance Online Publication.

20. Luhmann M, Hofmann W, Eid M, Lucas RE. Subjective well-being and adaptation to life events: A meta-analysis on differences between cognitive and affective well-being. J Pers Soc Psychol 2012; 102: 592-615.

21. Strauser DR, Lustig DC, Çiftçi A. Psychological well-being: Its relation to work personality, vocational identity, and career thoughts. J Psychol 2008; 142: 21-35.

22. Nielsen AS, Halamka JD, Kinkel RP. Internet portal use in an academic multiple sclerosis center. J Am Med Inform Assoc 2012; 19: 128-133.

23. Sinnenberg L, Buttenheim AM, Padrez K, Mancheno C, Ungar L, Merchant RM. Twitter as a tool for health research: A systematic review. Am J Public Health 2017; 107:143.

24. Wardell L, Hum S, Laizner AM, Lapierre Y. Multiple sclerosis patients’ interest in and likelihood of using online health-care services. Int J MS Care 2009; 11: 79-89.

25. Wicks P, Vaughan TE, Massagli MP. The Multiple Sclerosis Rating Scale, Revised (MSRS$\mathrm{R})$ :

Development, refinement, and psychometric validation using an online community. Health Qual Life Outcomes 2012; 10: 70.

26. Rossier P, Wade DT. The Guy's Neurological Disability Scale in patients with multiple sclerosis: 
a clinical evaluation of its reliability and validity. Clin Rehabil 2002; 16: 75-95.

27. Sharrack B, Hughes RA. The Guy's Neurological Disability Scale (GNDS): a new disability measure for multiple sclerosis. Mult Scler 1999; 5: 223-233.

28. Kolakowsky-Hayner SA, Kreutzer JS, Miner KD. Validation of the Service Obstacles Scale for

the traumatic brain injury population. NeuroRehabilitation 2000; 14: 151-158.

29. Holland JL, Johnston JA, Asama NF. The Vocational Identity Scale: A diagnostic and treatment tool. J Career Assess 1993; 1: 1-12.

30. Nitsch KP, Pedersen J, Miliotto A, Petersen B, Robbins S, Garcia A, Hoisington MA, Kimberly J;

Smiley J; Janikowski T. My Vocational Situation (MVS): Case example and psychometric review $\mathrm{Am}$

J Occup Ther 2017; 7102405010p1-7102405010p4.

31. Wenzel SL. The relationship of psychological resources and social support to job procurement self-efficacy in the disadvantaged. J Appl Soc Psychol 1993; 23: 1471-1497.

32. Bandura A. Self -efficacy: Towards a unifying theory of behavioural change. Psychol Rev 1977 ;

$84,191-215$

33. Honan CA, Brown RF, Hine DW, Vowels L, Wollin JA, Simmons RD, Pollard JD. The Multiple Sclerosis Work Difficulties Questionnaire. Mult Scler 2012; 18: 871-880

34. Scheier MF, Carver CS, Bridges MW. Distinguishing optimism from neuroticism (and trait anxiety, self-mastery, and self-esteem): A re-evaluation of the Life Orientation Test. J Pers Soc Psychol 1994; 67: 1063-1078. 
35. Hart SL, Vella L, Mohr DC. Relationships among depressive symptoms, benefit-finding, optimism, and positive affect in multiple sclerosis patients after psychotherapy for depression. Health Psychol 2008; 27: 230-238.

36. Kroenke K, Spitzer RL, Williams JBW. The PHQ-9: Validity of a brief depression severity measure. J Gen Intern Med 2001; 16: 606-613.

37. Amtmann D, Kim J, Chung H, Bamer AM, Askew RL, Wu S, Cook KF, Johnson KL. Comparing

CESD-10, PHQ-9, and PROMIS depression instruments in individuals with multiple sclerosis. Rehabil Psychol 2014; 59: 220-9.

38. Hind D, Kaklamanor D, Beever D, Webster R, Lee E, Barkham M, Cooper C. The assessment of depression in people with multiple sclerosis: A systematic review of psychometric validation studies. BMC Psychiatry 2016; 16:278.

39. Sjonnesen K, Berzins S, Fiest KM, Bulloch AG, Metz LM, Thombs BD, Patten SB. Evaluation of the 9-item Patient Health Questionnaire (PHQ-9) as an assessment instrument for symptoms of depression in patients with multiple sclerosis. Postgrad Med 2012; 124: 69-77.

40. White IR, Horton NJ, Carpenter J, Pocock SJ. Strategy for intention to treat analysis in randomised trials with missing outcome data. BMJ 2011; 342:d40

41. Ellis P. The essential guide to effect sizes. Cambridge UK: Cambridge University Press; 2010 42. Cohen J. A power primer. Psychol Bull 1992; 112: 155-159.

43. Faul F, Erdfelder E, Lang A-G, Buchner A. G*Power 3: A flexible statistical power analysis program for the social, behavioral, and biomedical sciences. Beh Res Meth 2007; 39: 175-191 
44. Ribbons K, Lea R, Tiedeman C, Mackenzie L, Lechner-Scott J. Ongoing increase in incidence and prevalence of multiple sclerosis in Newcastle, Australia: A 50-year study. Mult Scler 2016. Advance Online Publication.

45. Kalincik T. Multiple sclerosis relapses: Epidemiology, outcomes and management. A systematic review. Neuroepidemiology 2015; 44:199-214.

46. Schiavolin S, Leonardi M, Giovannetti AM, Antozzi C, Brambilla L, Confalonieri P, Mantegazza

R, Raggi A. Factors related to difficulties with employment in patients with multiple sclerosis: A review of 2002-2011 literature. J Rehabil Res 2013; 6:105-11.

47. Nutzi M, Trezzini B, Medici L, Schwegler U. Job matching: An interdisciplinary scoping study with implications for vocational rehabilitation counselling. Rehabil Psychol 2017; 62:4568.

48. Bolles R. What colour is your parachute? 2017: A practical guide for job-hunters and careerchangers. Danvers, MA: Ten Speed Press; 2016.

49. Doogan C, Playford E D. Supporting work for people with multiple sclerosis. Mult Scler 2014; 20: 646-50.

50. Hare M.A., Murphy, G.C. Predicting participation in interventions designed to promote job retention post-SCI. J Vocat Rehabil 2017; 46: 233-243.

51. Raggi A, Covelli V, Schiavolin S, Scaratti C, Leonardi M, Willems M. Work-related problems in multiple sclerosis: A literature review on its associates and determinants. Disabil Rehabil 2016; 38:936-44. 
Vocational rehabilitation and multiple sclerosis

52. Mathieu E, McGeechan K, Barratt A, Herbert R. Internet-based randomized controlled trials:

A

systematic review. J Am Med Inform Assoc 2012; 20:568-76.

53. Popp S, Schneider S. Attention placebo control in randomized controlled trials of psychosocial interventions: Theory and practice. Trials 2015; 16:150.

54. Foster G, Taylor SJ, Eldridge SE, Ramsay J, Griffiths CJ. Self-management education programmes by lay leaders for people with chronic conditions. Cochrane Database Syst Rev. 2007 Oct 17;(4):CD005108.

55. Burke J, Bezyak J, Fraser RT, Pete J, Ditchman N, Chan F. Employers'attitudes towards hiring and retaining people with disabilities: A review of the literature. AJRC 2013; 19: 21-38.

56. Chan F. Demand-side factors related to employment of people with disabilities: A survey of employers in the midwest region of the United States. J Occup Rehabil 2010; 20: 412-419.

57. Gupta A, Chong SH, Leong FTL. Development and validation of the Vocational Identity Measure. J Career Assess 2015; 23: 79-90.

58. Chung-Yi Chiu, Motl RW. (2015). Further validation of the Multiple Sclerosis SelfEfficacy Scale. Disabil Rehabil 2015; 37: 2429-2438. 
Table 1: Content and Structure of 'Work and MS' ${ }^{13}$

\begin{tabular}{|c|c|c|}
\hline \multicolumn{2}{|c|}{ Module } & \multirow{2}{*}{$\begin{array}{l}\text { Topics covered } \\
\text { - Overview of 'Work and MS' aims and objectives } \\
\text { - } \quad \text { Email details of moderator }\end{array}$} \\
\hline 1 & Introduction & \\
\hline 2 & Job facts & $\begin{array}{l}\text { - } \quad \text { Facts about MS and employment } \\
\text { - Work benefits (physical, psychological) } \\
\text { - } \quad \text { Managing work barriers }\end{array}$ \\
\hline 3 & Finding work & $\begin{array}{l}\text { - } \text { Characteristics of the Australian job market } \\
\text { - } \text { Researching potential employers } \\
\text { - } \text { Further study options } \\
\text { - } \text { Disability employment services in Australia } \\
\text { - Maintaining psychological health } \\
\text { - } \text { Case study }\end{array}$ \\
\hline 4 & $\begin{array}{l}\text { Resume writing \& interview } \\
\text { preparation }\end{array}$ & $\begin{array}{l}\text { - Determining 'job-fit' } \\
\text { - Structure and content of cover letters and resumes } \\
\text { - Interview preparation }\end{array}$ \\
\hline 5 & Job interview do's \& don'ts & $\begin{array}{l}\text { - Answering difficult interview questions } \\
\text { - Asking interview questions } \\
\text { - Interview follow-up }\end{array}$ \\
\hline 6 & Maintaining work & $\begin{array}{l}\text { - } \text { Disclosure of MS } \\
\text { - Managing MS symptoms in the workplace } \\
\text { - } \text { Managing the work environment: work arrangements and job role } \\
\text { - Case study }\end{array}$ \\
\hline 7 & Career development & $\begin{array}{l}\text { - Maintaining and developing work skills } \\
\text { - Finding a career mentor }\end{array}$ \\
\hline
\end{tabular}


Table 2: Sociodemographic and MS details $(N=95)$

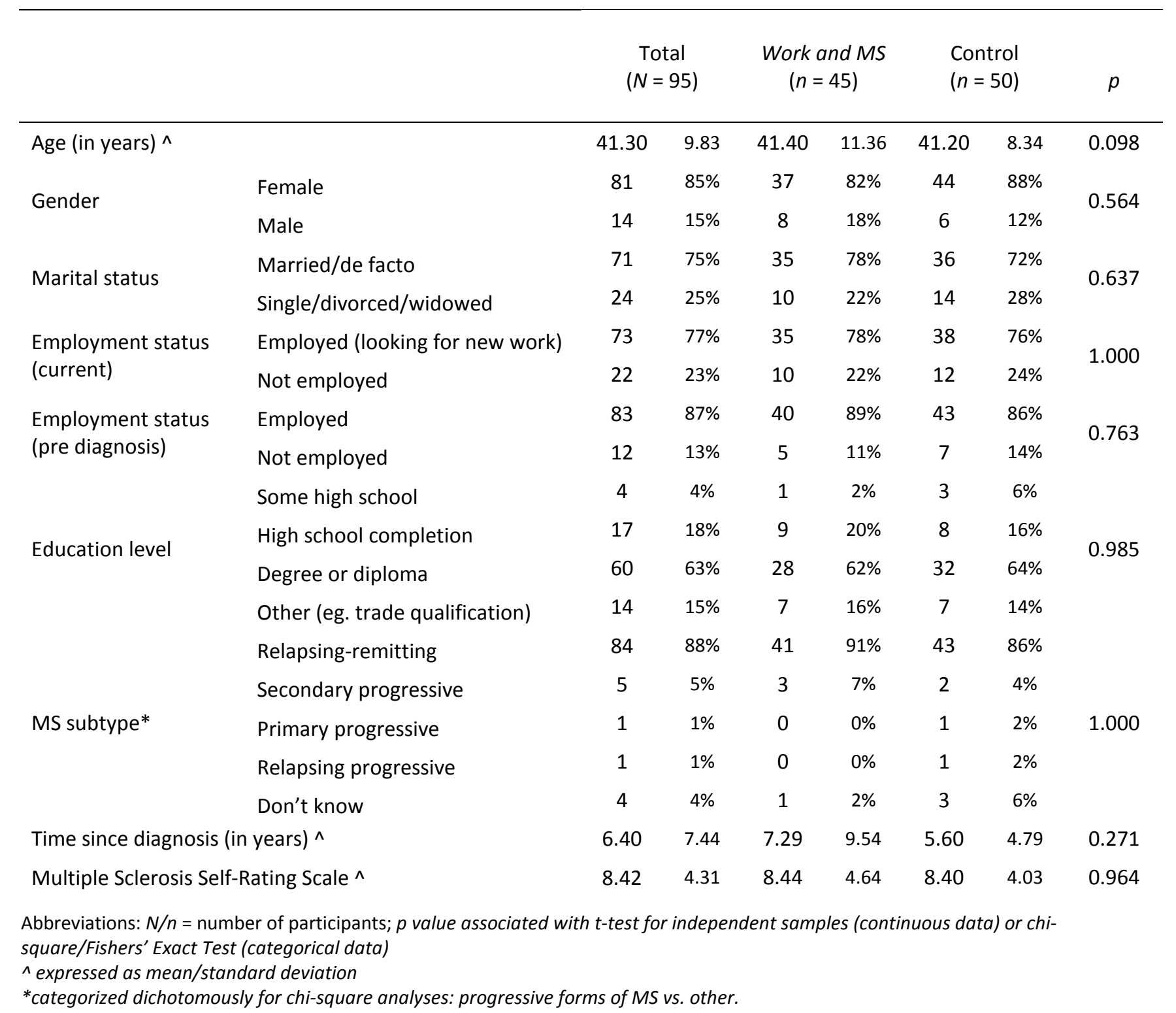


Table 3: Correlational matrix of continous variables (at baseline)

\begin{tabular}{|c|c|c|c|c|c|c|c|c|c|c|c|c|c|}
\hline & & & 1 & 2 & 3 & 4 & 5 & 6 & 7 & 8 & 9 & 10 & 11 \\
\hline & & & $r$ & $0.49 * *$ & $0.26 * *$ & -0.05 & 0.18 & -0.03 & 0.04 & -0.08 & -0.08 & -0.02 & -0.12 \\
\hline \multirow[t]{3}{*}{1} & \multirow{3}{*}{\multicolumn{2}{|c|}{ Age }} & $p$ & 0.00 & 0.01 & 0.65 & 0.09 & 0.76 & 0.71 & 0.45 & 0.43 & 0.87 & 0.24 \\
\hline & & & $n$ & 95 & 95 & 95 & 94 & 90 & 95 & 94 & 95 & 95 & 94 \\
\hline & & & & - & 0.03 & 0.12 & 0.11 & -0.08 & -0.00 & -0.09 & -0.04 & -0.10 & $-0.22 *$ \\
\hline \multirow[t]{3}{*}{2} & \multirow{3}{*}{\multicolumn{2}{|c|}{ Time since diagnosis }} & & & 0.81 & 0.25 & 0.28 & 0.47 & 0.98 & 0.39 & 0.74 & 0.34 & 0.04 \\
\hline & & & & & 95 & 95 & 94 & 90 & 95 & 94 & 95 & 95 & 94 \\
\hline & & & & & - & 0.18 & $-0.27^{* *}$ & -0.08 & $0.23 *$ & $0.40 * *$ & $0.38^{* *}$ & $0.49 * *$ & $0.28 *$ \\
\hline \multirow[t]{3}{*}{3} & MSRS-R & & & & & 0.08 & 0.01 & 0.46 & 0.03 & 0.00 & 0.00 & 0.00 & 0.01 \\
\hline & & & & & & 95 & 94 & 90 & 95 & 94 & 95 & 95 & 94 \\
\hline & & & & & & - & $-0.30^{* *}$ & $-0.22^{*}$ & $0.21 *$ & $0.26^{*}$ & $0.27^{*}$ & 0.15 & $0.22 *$ \\
\hline \multirow[t]{3}{*}{4} & sos & & & & & & 0.00 & 0.04 & 0.05 & 0.01 & 0.01 & 0.16 & 0.03 \\
\hline & & & & & & & 94 & 90 & 95 & 94 & 95 & 95 & 94 \\
\hline & & & & & & & - & $0.51^{* *}$ & -0.16 & $-0.56 * *$ & $-0.44^{* *}$ & $-0.24 *$ & $-0.38 * *$ \\
\hline \multirow[t]{3}{*}{5} & MVS & & & & & & & 0.00 & 0.13 & 0.00 & 0.00 & 0.02 & 0.00 \\
\hline & & & & & & & & 90 & 94 & 93 & 94 & 94 & 94 \\
\hline & & & & & & & & - & $-0.27^{* *}$ & $-0.38 * *$ & $-0.41 * *$ & -0.12 & $-0.23 *$ \\
\hline \multirow[t]{3}{*}{6} & JSES & & & & & & & & 0.01 & 0.00 & 0.00 & 0.25 & 0.03 \\
\hline & & & & & & & & & 90 & 89 & 90 & 90 & 90 \\
\hline & & & & & & & & & - & $0.45^{* *}$ & $0.51^{* *}$ & $0.38^{* *}$ & $0.50 * *$ \\
\hline \multirow[t]{3}{*}{7} & MSWDQ & work home balance & & & & & & & & 0.00 & 0.00 & 0.00 & 0.00 \\
\hline & & & & & & & & & & 94 & 95 & 95 & 94 \\
\hline & & & & & & & & & & - & $0.67^{* *}$ & $0.47^{* *}$ & $0.52^{* *}$ \\
\hline \multirow[t]{3}{*}{8} & & self-esteem & & & & & & & & & 0.00 & 0.00 & 0.00 \\
\hline & & & & & & & & & & & 94 & 94 & 93 \\
\hline & & & & & & & & & & & - & $0.56^{* *}$ & $0.49 * *$ \\
\hline \multirow[t]{3}{*}{9} & & relationships & & & & & & & & & & 0.00 & 0.00 \\
\hline & & & & & & & & & & & & 95 & 94 \\
\hline & & & & & & & & & & & & - & $0.31^{* *}$ \\
\hline \multirow[t]{3}{*}{10} & & accessibility & & & & & & & & & & & 0.00 \\
\hline & & & & & & & & & & & & & 94 \\
\hline & & & & & & & & & & & & & - \\
\hline 11 & & finances & & & & & & & & & & & \\
\hline 12 & & supports & & & & & & & & & & & \\
\hline
\end{tabular}

13 LOT-R

14 PHQ-9

* correlation is significant at the 0.05 level (2-tailed) ** correlation is significant at the 0.01 level (2-tailed)

Table 4: Pre-post scores and associated effect sizes after accessing Work and MS (completers only)

$\begin{array}{lll}\text { Measure } \quad \text { Work and MS Control } & \text { Analyses }\end{array}$




\begin{tabular}{|c|c|c|c|c|c|c|c|c|c|c|}
\hline & Time & $N$ & Mean & SD & $N$ & Mean & SD & $t$ & $d f$ & $p$ \\
\hline \multicolumn{11}{|l|}{ Primary outcomes } \\
\hline \multirow{2}{*}{$\begin{array}{l}\text { My Vocational Situation } \\
\text { vocational identity }\end{array}$} & 1 & 45 & 9.38 & 4.42 & 49 & 10.27 & 4.49 & \multirow{2}{*}{-2.68} & \multirow{2}{*}{70} & \multirow{2}{*}{0.009} \\
\hline & 2 & 28 & 10.54 & 5.05 & 44 & 9.11 & 4.56 & & & \\
\hline \multirow{2}{*}{ Job Procurement Self-efficacy } & 1 & 43 & 65.42 & 18.78 & 47 & 58.11 & 18.89 & \multirow{2}{*}{-1.24} & \multirow{2}{*}{62} & \multirow{2}{*}{0.221} \\
\hline & 2 & 24 & 70.50 & 14.86 & 43 & 56.65 & 18.47 & & & \\
\hline \multicolumn{11}{|l|}{ Secondary outcomes } \\
\hline \multirow{3}{*}{$\begin{array}{l}\text { MS Work Difficulties } \\
\text { work-home balance }\end{array}$} & 1 & 45 & 37.41 & 26.33 & 50 & 33.67 & 26.67 & \multirow{2}{*}{1.29} & \multirow{2}{*}{68} & \multirow{2}{*}{0.203} \\
\hline & 2 & 27 & 37.04 & 32.05 & 43 & 37.02 & 26.25 & & & \\
\hline & 1 & 45 & 37.96 & 27.44 & 49 & 40.31 & 26.81 & \multirow{2}{*}{2.47} & \multirow[b]{2}{*}{67} & \multirow[b]{2}{*}{0.016} \\
\hline self-esteem & 2 & 27 & 32.41 & 25.25 & 43 & 44.38 & 24.24 & & & \\
\hline \multirow{2}{*}{ relationships } & 1 & 45 & 26.67 & 24.33 & 50 & 26.83 & 20.71 & \multirow[b]{2}{*}{2.44} & \multirow[b]{2}{*}{67} & \multirow[b]{2}{*}{0.017} \\
\hline & 2 & 27 & 22.84 & 21.63 & 42 & 31.94 & 23.35 & & & \\
\hline \multirow{2}{*}{ accessibility } & 1 & 45 & 16.67 & 19.26 & 50 & 19.63 & 25.03 & \multirow[b]{2}{*}{1.57} & \multirow[b]{2}{*}{68} & \multirow[b]{2}{*}{0.120} \\
\hline & 2 & 27 & 12.96 & 18.98 & 43 & 18.31 & 23.91 & & & \\
\hline \multirow{2}{*}{ finances } & 1 & 45 & 60.14 & 25.81 & 49 & 57.27 & 28.88 & \multirow{2}{*}{2.14} & \multirow{2}{*}{68} & \multirow{2}{*}{0.036} \\
\hline & 2 & 27 & 52.78 & 24.66 & 43 & 63.22 & 22.50 & & & \\
\hline \multirow{2}{*}{ supports } & 1 & 45 & 32.89 & 24.48 & 50 & 30.00 & 24.89 & \multirow{2}{*}{1.27} & & \\
\hline & 2 & 27 & 31.48 & 28.58 & 41 & 29.88 & 25.92 & & 66 & 0.207 \\
\hline Life Orientation Test Revised & 1 & 45 & 13.31 & 5.85 & 50 & 13.66 & 5.66 & 049 & 11 & 0630 \\
\hline Lile Unemation tess nevised & 2 & 20 & 13.50 & 6.64 & 23 & 12.09 & 7.15 & 0.45 & 41 & 0.050 \\
\hline Patient Health Questionnaire & 1 & 45 & 9.11 & 6.31 & 50 & 8.48 & 5.63 & 18 & 67 & רגב ח \\
\hline 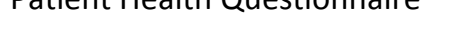 & 2 & 26 & 7.96 & 6.47 & 43 & 8.53 & 5.35 & 1.10 & 07 & 0.242 \\
\hline
\end{tabular}

Note: effect sizes standardized so that a positive value indicate greater gains in psychological functioning for 'Work and MS' intervention participants in comparison to wait-list controls. The direction of the MSWDQ and PHQ were re-scaled to conform to this rule. 